\title{
HE 0047-1756: A new gravitationally lensed double QSO^
}

\author{
L. Wisotzki ${ }^{1,2}$, P. L. Schechter ${ }^{3}$, H.-W. Chen ${ }^{3}$, D. Richstone ${ }^{4}$, K. Jahnke ${ }^{1}$, S. F. Sánchez ${ }^{1}$, and D. Reimers ${ }^{5}$ \\ 1 Astrophysikalisches Institut Potsdam, An der Sternwarte 16, 14482 Potsdam, Germany \\ e-mail: lwisotzki@aip.de \\ 2 Universität Potsdam, Am Neuen Palais 10, 14469 Potsdam, Germany \\ 3 Center for Space Research, Massachusetts Institute of Technology, Cambridge, MA 02139, USA \\ ${ }^{4}$ Dept. of Astronomy, University of Michigan, 830 Dennison, 501 East University Avenue, Ann Arbor, MI 48109, USA \\ 5 Hamburger Sternwarte, Universität Hamburg, Gojenbergsweg 112, 21029 Hamburg, Germany
}

Received 19 March 2004 / Accepted 4 April 2004

\begin{abstract}
The quasar HE 0047-1756, at $z=1.67$, is found to be split into two images 1 .' 44 apart by an intervening galaxy acting as a gravitational lens. The flux ratio for the two components is roughly $3.5: 1$, depending slightly upon wavelength. The lensing galaxy is seen on images obtained in the $i(800 \mathrm{~nm})$ and $K_{\mathrm{s}}$ bands $(2.1 \mu \mathrm{m})$; there is also a nearby faint object which may be responsible for some shear. The spectra of the two quasar images are nearly identical, but the emission line ratio between the two components scale differently from the continuum. Moreover, the fainter component has a bluer continuum slope than the brighter one. We argue that these small differences are probably due to microlensing. There is evidence for a partial Einstein ring emanating from the brighter image toward the fainter one.
\end{abstract}

Key words. quasars: individual: HE 0047-1756 - quasars: general - gravitational lensing

\section{Introduction}

Every quasar lensed by an intervening galaxy presents opportunities to study the structure of quasars, lensing galaxies and the intervening intergalactic medium. Every year sees the discovery of new systems ${ }^{1}$ and followup studies that take advantage of the peculiar circumstances of each new system.

In this paper we report the discovery a new gravitational lens, producing two images of the quasar HE 0047-1756. It is the second system found in the course of an ongoing survey for the lensing of quasars using the Magellan consortium 6.5-m telescopes on Cerro Las Campanas (Wisotzki et al. 2002). We present results from optical and near-IR images obtained with these telescopes, plus integral field data obtained at Calar Alto observatory.

\section{Observations}

\subsection{Identification}

HE 0047-1756 was first discovered in the course of the Hamburg/ESO bright quasar survey (HES; Wisotzki et al. 2000). The survey uses digitised objective prism spectra to

Send offprint requests to: L. Wisotzki, e-mail: lutz@aip.de

* Based in part on observations obtained with the Baade 6.5 -m telescope of the Magellan Consortium. Based in part on observations collected at the German-Spanish Astronomical Center, Calar Alto, operated jointly by Max-Planck-Institut für Astronomie and Instituto de Astrofísica de Andalucia (CSIC).

${ }^{1}$ Nine discoveries were reported in the 2003 postings on the $\mathrm{xxx}$. arXiv. org preprint server. perform a largely automated QSO search covering the entire southern extragalactic sky. HE 0047-1756 was identified in this survey as a high probability QSO candidate with redshift $z=1.66$. However, its apparent magnitude of $B_{\mathrm{J}}=16.98$ (measured in the Digital Sky Survey linked to HES photometry) put it below the flux threshold for systematic follow-up spectroscopy in that particular field, and the object remained listed in the archive as a high probability QSO candidate with formal redshift confirmation still pending.

The Magellan lens survey targets apparently bright quasars at substantial redshifts. Because of magnification bias (Turner 1980), such objects have an enhanced probilility of being affected by lensing. Although HE 0047-1756 was not yet a confirmed QSO, the nature of the object was thought to be sufficiently well determined to include it, together with several similar cases, in the target list.

\subsection{Optical imaging}

We obtained high resolution optical images at the Baade $6.5 \mathrm{~m}$ (Magellan I) telescope on 14 Dec. 2001, equipped with the Magellan Instant Camera (MagIC) and a $2 \mathrm{k} \times 2 \mathrm{k}$ CCD. The image scale at the f/11 Nasmyth focus was 0.'0692 per pixel. The active optics system (Schechter et al. 2003) updated the focus and translation of the secondary and the twelve most flexible elastic modes of the primary mirror at half minute intervals. A first $r^{\prime}$ band "snapshot" of $30 \mathrm{~s}$ immediately showed clearly that the object was double, consisting of at least two point sources. Three further images were obtained, one each in the SDSS $u^{\prime}$, 

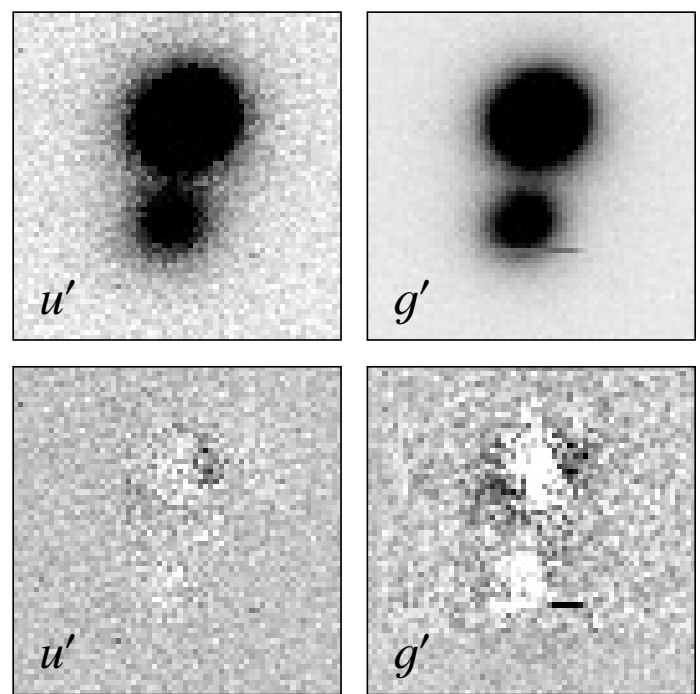
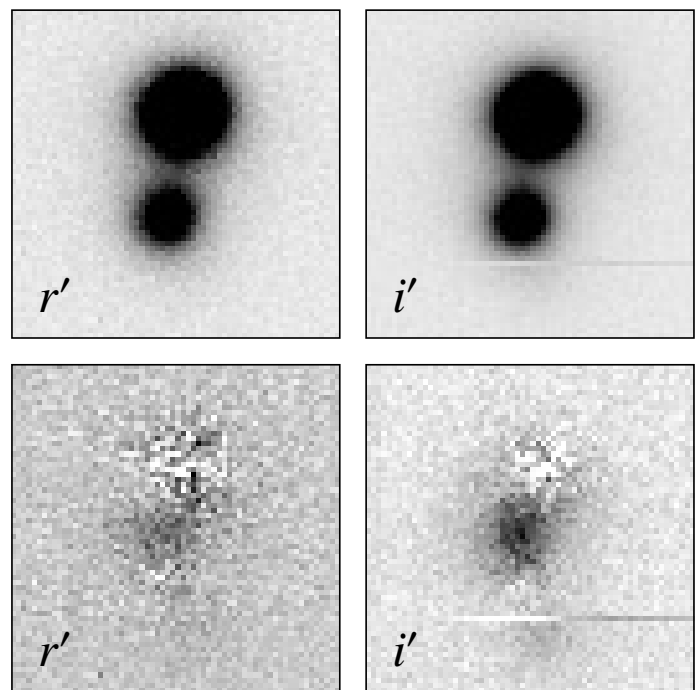
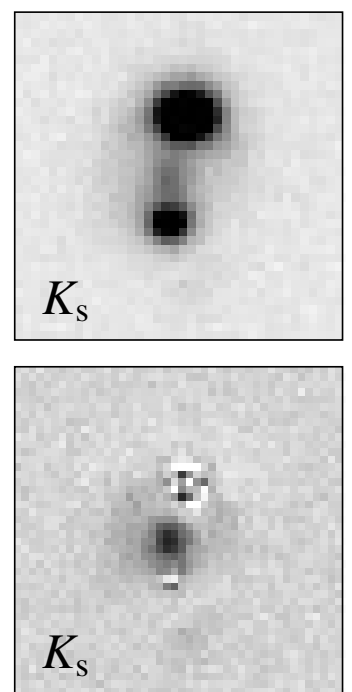

Fig. 1. Top row: magellan optical and near-infrared images of the double QSO, in standard astronomical orientation (North up, East left). The size of each image is 4"'4. The photometric bands are denoted in the lower left corners. Some low-level pickup noise is present in the $u$ and $g$ images. Bottom row: corresponding point source-subtracted images, based on the best-fit solutions in each band.

$g^{\prime}$ and $i^{\prime}$ bands (for simplicity, we shall refer to these bands as ugri in the following). The former was exposed for $300 \mathrm{~s}$, the latter two for $120 \mathrm{~s}$ each. The effective seeing was around 0 .' $^{\prime} 6$, implying that the images were highly oversampled. The night was not photometric, however, and we therefore did not attempt to calibrate the data with standard stars. Following the usual data reduction steps of debiasing and flatfielding, we extracted postage stamp images around the QSO which are shown in Fig. 1. The fact that both components are bright in the $u$ band is by itself strongly suggestive that each point source shows a quasar. We denote the brighter, northern component as A, and the fainter one as B.

Relative positions and magnitudes of the individual components were measured using the program GALFIT kindly provided by Dr. C. Y. Peng (described in Peng et al. 2002) which performs simultaneous fits of an arbitrary number of point sources and extended components to an image. The point spread function (PSF) was obtained from the only other reasonably bright star within the field of view, about $45^{\prime \prime} \mathrm{NW}$ from the QSO. Fitting and subtracting only two scaled PSFs gave residuals consistent with zero only for the short wavelength images. By contrast, the PSF-subtracted $r$ and $i$ images show clearly what appears to be the lensing galaxy. Including an extended component at the approximate position of the galaxy, modelled by an de Vaucouleurs law, considerably improved the fit (total $\chi^{2}=1.34$ per degree of freedom). The shape of the lensing galaxy, however, is not well constrained in the $i$ band image because of blending with the $\mathrm{B}$ component. The astrometric and photometric results of the fits are given in Tables 1 and 2. The separation between A and B is $1^{\prime \prime} .44$, at a position angle of $261^{\circ}$ measured from A. The coordinates of component $\mathrm{A}$, measured relative to three nearby stars listed in the USNOB catalogue, is RA $=00^{\mathrm{h}} 50^{\mathrm{m}} 27^{\mathrm{s}} .83$, Dec $=-17^{\circ} 40^{\prime} 8^{\prime \prime} .8$ $\left( \pm 0{ }^{\prime} 1\right)$.

The presence of a galaxy within a few tenths of an arcsecond of where one would have expected it is compelling evidence for the lens interpretation. The faintness of the lensing
Table 1. Differential astrometry for HE 0047-1756, based on the GALFIT results for the $i$ and $K_{\mathrm{s}}$ band images. Component A is the brighter of the two QSO components. The quoted error bars are only the internal errors given by the fitting routine. The last object is the faint blob southwest of component B.

\begin{tabular}{lcc}
\hline \hline Component/band & $\begin{array}{c}\Delta \alpha \\
{[\operatorname{arcsec}]}\end{array}$ & $\begin{array}{c}\Delta \delta \\
{[\operatorname{arcsec}]}\end{array}$ \\
\hline $\mathrm{A}$ & $0.000 \pm 0.000$ & $0.000 \pm 0.000$ \\
$\mathrm{~B}(i)$ & $0.226 \pm 0.001$ & $-1.418 \pm 0.001$ \\
$\mathrm{~B}\left(K_{\mathrm{s}}\right)$ & $0.230 \pm 0.001$ & $-1.433 \pm 0.001$ \\
$\mathrm{G}(i)$ & $0.227 \pm 0.007$ & $-0.865 \pm 0.013$ \\
$\mathrm{G}\left(K_{\mathrm{s}}\right)$ & $0.267 \pm 0.008$ & $-0.881 \pm 0.009$ \\
blob & $-0.07 \pm 0.15$ & $-2.36 \pm 0.15$ \\
\hline
\end{tabular}

Table 2. Differential photometry for the fainter QSO component and the lensing galaxy. The numbers are in magnitudes relative to component $\mathrm{A}$. The last object is again the faint blob southwest of B.

\begin{tabular}{lccccc}
\hline \hline Component & $u$ & $g$ & $r$ & $i$ & $K_{\mathrm{s}}$ \\
\hline A & 0.00 & 0.00 & 0.00 & 0.00 & 0.00 \\
B & 1.31 & 1.39 & 1.49 & 1.50 & 1.46 \\
G & & & 2.23 & 2.10 & 1.44 \\
blob & & & & 4.8 & \\
\hline
\end{tabular}

galaxy in the filters blueward of $i$ argues for a relatively high redshift $(z \gtrsim 0.6)$ for the lensing galaxy.

The $i$ band image shows also a second, very faint object southwest of component $\mathrm{B}$. This is more obviously seen in the residual image (after PSF and galaxy subtraction) of Fig. 2. Approximate position and magnitude estimates $( \pm 0.3 \mathrm{mag})$ for this faint blob have been added to Tables 1 and 2 . 

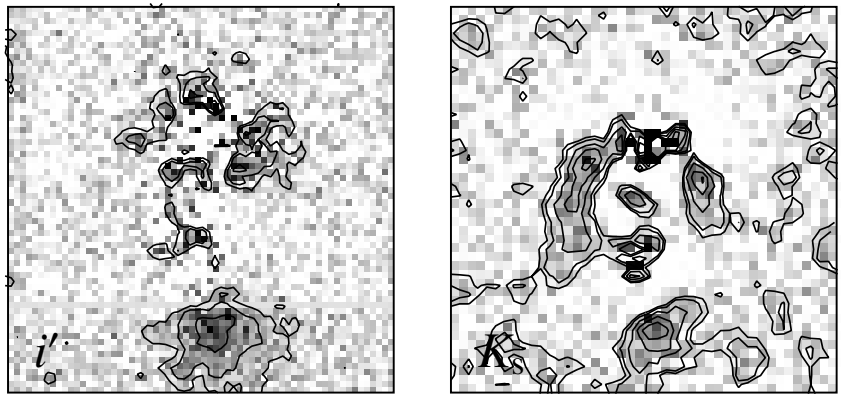

Fig. 2. Residuals after subtraction of point sources and the lensing galaxy, in the $i(l e f t)$ and $K_{\mathrm{s}}(r i g h t)$ bands. Notice the almost complete Einstein ring in the near-infrared image, not present in $i$.

\subsection{Near IR imaging}

A series of nine dithered 1.5 min exposures of HE 0047-1756 was obtained on 2002 November 13 in the $K_{\mathrm{s}}$ filter using CassiCam on the Baade $6.5-\mathrm{m}$ telescope in non-photometric conditions. The $F W H M$ in the stacked image was 0 ' 32 , the pixels are $0{ }^{\prime} 115$ on a side. The lensing galaxy is clearly visible in Fig. 1. Simultaneous PSF fitting to the quasar and the galaxy using GALFIT yielded another set of relative positions and fluxes, also listed in Tables 1 and 2. Because of the narrower PSF, the $K_{\mathrm{s}}$ band constrains the shape of the lensing galaxy much better than the optical data. As seen in the PSF-subtracted image in Fig. 1, the lensing galaxy is very nearly round. The fitted ellipticity is $a / b=0.81 \pm 0.05$, the half-light radius is $0{ }^{\prime} 52 \pm 00^{\prime} 04$. The differences between the near IR and optical are mostly due to uncertainty in the instrument scale. The position of the lensing galaxy is close to (but not exactly on) the line connecting components A and B, at a distance of 0.' 89 from A and 0.55 from B.

After subtraction of both PSFs and a model for the lensing galaxy, the residual image shows significant nonzero flux, most of which is in form of arc-like features protruding from the brighter image and stretching toward the fainter one (cf. Fig. 2). There may also be similar, but fainter arcs emanating from component $\mathrm{B}$. These features are strongly suggestive of an at least partial Einstein ring due to the deformed QSO host galaxy. The $i$ band image does not show any arcs, consistent with the fact that the source is at the redshift of the QSO, in which case the $i$ band is located below the $4000 \AA$ break in the source restframe. Figure 2 also shows the companion object southwest of component $\mathrm{B}$, but only very faintly.

\subsection{Long slit spectroscopy}

Long slit spectra were obtained with the Boller and Chivens spectrograph on the Baade 6.5-m telescope on 2001 December 21. The 1" slit was oriented North-South, passing through both quasar images. Two spectra, of $300 \mathrm{~s}$ and $600 \mathrm{~s}$, were taken. The two components were spatially well separated. Both quasar images show emission lines at $4130 \AA$ and $5099 \AA$ that we identified as C IV $\lambda 1550$ and C III] $\lambda 1909$ at a redshift of 1.67, confirming the objective prism redshift. The redshift difference $z_{\mathrm{B}}-z_{\mathrm{A}}$ corresponds to a velocity difference
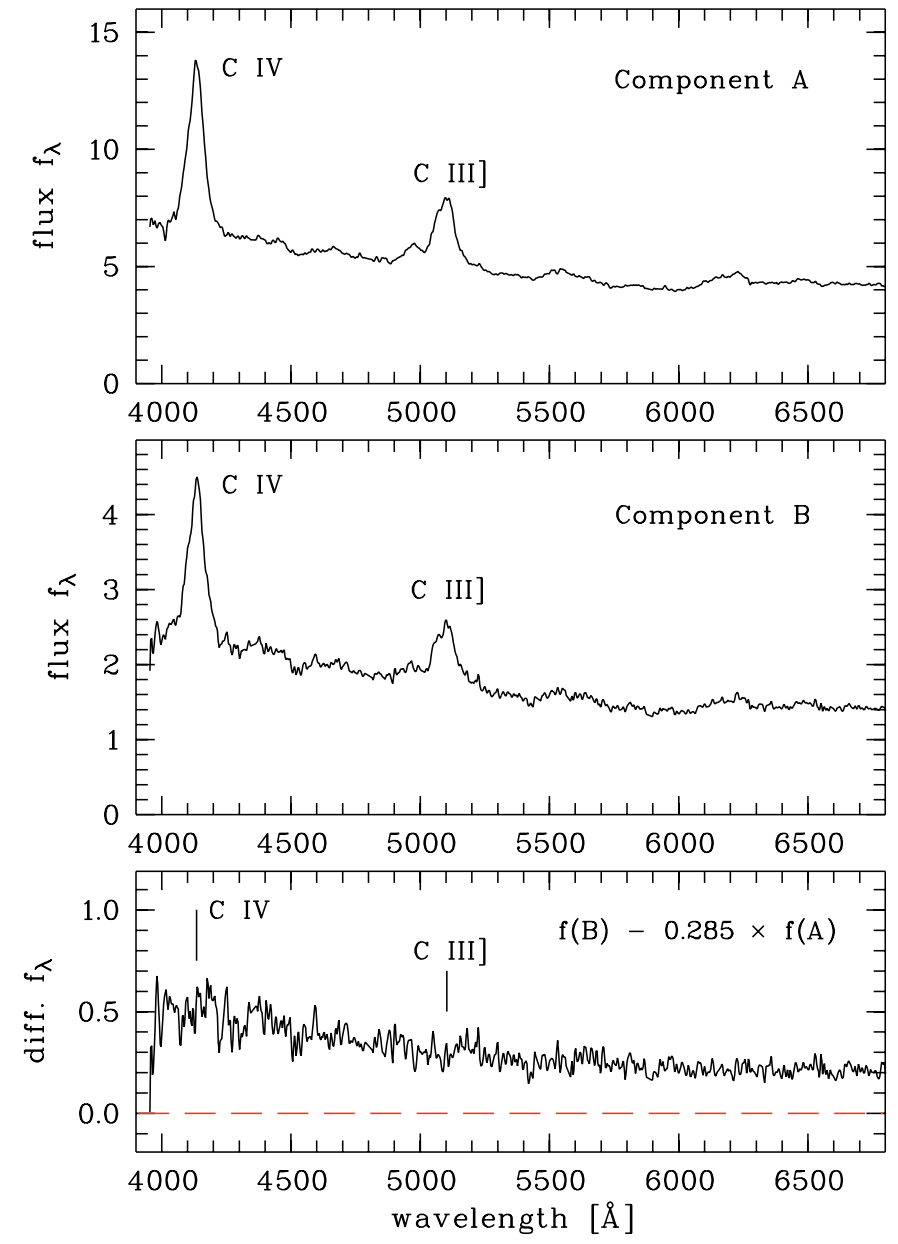

Fig. 3. Top and middle panel: extracted and calibrated PMAS spectra. Bottom panel: scaled difference spectrum showing the putative microlensing component. Main emission lines have been marked.

of less that $30 \mathrm{~km} \mathrm{~s}^{-1}$, in excellent agreement with the lensing hypothesis.

\subsection{Integral field spectrophotometry}

We observed the double QSO on 2002 September 4 with the Potsdam Multi-Aperture Spectrophotometer PMAS (Kelz et al. 2003), mounted on the Calar Alto 3.5-m telescope. The array of $16 \times 16$ spatial picture elements of each $0.5 \times 00^{\prime} 5$ was adequate both to cover the object and to resolve the images. The effective seeing was 1'.2, and the night was photometric. Notice that since the PMAS microlens array reimages the exit pupil of the telescope, there are no geometrical losses due to incomplete filling of the focal plane. The data were reduced with the IDL-based software package P3d (Becker 2002). The reduction consists of standard steps such as debiasing and flatfielding using twilight exposures, and dedicated routines such as tracing and extracting the spectra of individual fibres and reassembling the data in form of a three-dimensional data cube. The spectra were extracted with the iterative fitting method described by Wisotzki et al. (2003), which also accounts for differential atmospheric refraction. 
In the spectra, shown in Fig. 3, we again see C IV and C III] as well as Fe II features at $5600 \AA$ and $6300 \AA$. There was insufficient signal to extract a spectrum of the lensing galaxy. The relative strengths of the emission lines are very similar in the two components, scaling by a factor of $0.285(1.36 \mathrm{mag})$ from A to B. Subtracting the brighter component, scaled by this factor, from the fainter one eliminates any trace of the emission lines, thus also the line profiles are indistinguishable. However, the continuum does not subtract out by the same factor, and there is significant residual flux left in component B. Could this excess be due to contamination by the lensing galaxy? From the spectral shape of the excess, this would be highly unlikely: we know from Table 2 that the lensing galaxy is much redder than the QSO, whereas the excess continuum is markedly blue. Equally, a contamination from the faint blob south of B can be excluded given its angular separation; it furthermore does not show up in the $g$ band image.

The presence of blue residual flux argues either for differential microlensing between the two images (very similar to what is observed in HE 1104-1805, Wisotzki et al. 1993), or differential temporal variation in the underlying continuum, which is emitted at two different epochs due to time delay effects. We have examined the Magellan long-slit spectrum taken 256 days earlier and find a similar difference with the same sign, although the narrow slit precludes a quantitative relative spectrophotometry. Nevertheless, the fact that the flux difference has the same sign at both spectroscopic epochs favours the microlensing interpretation.

\section{Models}

A model which works quite well for many lenses is a singular isothermal sphere with an external shear, as might be generated by the tide from a neighboring galaxy or cluster. The two dimensional projected potential (e.g., Kochanek 1991) is given by

$\phi(r)=b r+\frac{\gamma}{2} r^{2} \cos \left(\phi-\phi_{\gamma}\right)$

where $b$ is the diameter of the isothermal sphere in arcseconds, $r$ and $\phi$ are the radial and angular parts, respectively, of the angular position on the sky with respect to the galaxy, and $\phi_{\gamma}$ is the position angle of the shear, measured E of N. Given our sign convention, a bar or tidal perturber would be at right angles to $\phi_{\gamma}$. Fitting this model to the positions in Table 1 and to the emission line flux ratio, we get $b=0.770, \gamma=0.0637$ and $\phi_{\gamma}=104^{\circ} 9$, with a source position $(\Delta \alpha, \Delta \delta)=\left(-0 \prime^{\prime} 001,0{ }^{\prime}{ }^{\prime} 184\right)$ relative to the measured position for the lens. As the number of free parameters is equal to the number of constraints, the fit is perfect.

The position angle of the shear would imply an object producing a tide at a position angle of 14.9 or $194^{\circ} .9$. The faint blob to the south of the fainter image lies at PA 192.8. The amplitude of the shear is not large. Taking the blob to be a singular isothermal sphere at a distance 1 1"52 from the lensing galaxy, the inferred Einstein radius for the blob would be 0!'193. This is a factor of 4 smaller than for the lens, implying a luminosity a factor of 16 smaller if the Faber-Jackson or Tully-Fisher relation were appropriate. It is consistent with our measurement (Table 2) of $i_{\text {blob }}-i_{\text {lens }}=2.7 \pm 0.3$.

The magnifications for the two images is 2.1 for the fainter (negative parity) image and 7.3 for the brighter (positive parity) image. The differential time delay is a strong function of the unknown redshift of the lensing galaxy; it is 32.1 days for $z_{\text {lens }}=0.6$ and increases proportionally to $\left(1+z_{\text {lens }}\right)$.

\section{Conclusions}

The newly discovered double QSO HE 0047-1756 displays several properties that make it a showpiece gravitationally lensed object rather than a binary QSO: the identical (within $\pm 30 \mathrm{~km} \mathrm{~s}^{-1}$ ) redshifts of the two quasar images; the similar emission line profiles; the detection of a galaxy located between the two QSO components; and the tentative discovery of a partial Einstein ring.

The only apparent deviation from the simple notion of two identical images is the slight difference in spectral slopes. While spectral variability and light travel time effects cannot be excluded as an explanation, we argue that the spectral differences are more likely due to differential microlensing on the compact continuum source of the quasar. Spectrophotometric monitoring is required to resolve the issue unambiguously.

The existence of a prominent NIR Einstein ring makes this object particularly interesting for several applications. Firstly, the geometry of the distortions can be used to obtain additional constraints on the lens potential, overcoming the shortage of constraints of double-image lenses. Secondly, the fact that the distorted quasar host galaxy is also highly magnified can be exploited to study the properties of the quasar host. HST imaging data will be required to accomplish these tasks.

Acknowledgements. The Hamburg/ESO Survey was supported as ESO key programme 02-009-45K (145.B-0009). MagIC was built with help from a gift by Raymond and Beverly Sackler to Harvard University and a US NSF grant, AST99-77535, to MIT. PMAS was partly financed by BMBF/Verbundforschung unter 053PA414/1 and 05AL9BA1/9. PLS gratefully acknowleges the support of the US NSF under award AST-0206010. SFS ackowledges financial support provided through the European Community's Human Potential Program under contract HPRN-CT-2002-00305, Euro3D RTN. KJ and LW acknowledge a DFG travel grant under Wi 1369/12-1.

\section{References}

Becker, T. 2002, Ph.D. Thesis, Universität Potsdam

Kelz, A., Roth, M. M., \& Becker, T. 2003, Proc. SPIE, 4841, 1057

Kochanek, C. S. 1991, ApJ, 373, 354

Peng, C. Y., Ho, L. C., Impey, C. D., \& Rix, H. 2002, AJ, 124, 266

Schechter, P. L., Burley, G. S., Hull, C. L., et al. 2003, Proc. SPIE, 4837, 619

Turner, E. L. 1980, ApJ, 242, L135

Wisotzki, L., Becker, T., Christensen, L., et al. 2003, A\&A, 408, 455

Wisotzki, L., Christlieb, N., Bade, N., et al. 2000, A\&A, 358, 77

Wisotzki, L., Köhler, T., Kayser, R., \& Reimers, D. 1993, A\&A, 278, L15

Wisotzki, L., Schechter, P. L., Bradt, H. V., Heinmüller, J., \& Reimers, D. 2002, A\&A, 395, 17 